\author{
Jean-Louis Haquette ${ }^{*}$ \\ Université de Reims Champagne Ardenne - CRIMEL (EA 3311)
}

\title{
PAOLO ROLLI \& VOLTAIRE: MODÈLES LITTÉRAIRES ET CONTESTATION CRITIQUE
}

\begin{abstract}
Il dibattito critico che oppone Voltaire e Paolo Rolli testimonia il proficuo scambio transnazionale e la diffusione di modelli letterari nel Settecento europeo. In questa circolazione di valori le traduzioni svolgono un ruolo centrale. La complessa storia editoriale di testi e commenti redatti e tradotti in inglese, francese e italiano fa emergere posizioni opposte sulla poesia epica e, più in generale, sulla letteratura. Le versioni dei due letterati del celebre soliloquio di Amleto, d'altro canto, sottolineano concezioni diverse della traduzione, ma confluiscono alla fine nel riconoscimento del genio sublime della lingua shakespeariana.

Parole chiave: Paolo Rolli, Voltaire, soliloquio di Amleto, dibattito critico transnazionale, modelli letterari, concezioni della traduzione.
\end{abstract}

Au siècle des Lumières, si les littératures nationales affirment leur identité, elles le font au sein de la République européennes des lettres, communauté d'échanges dont les traductions sont une composante importante (cf. Beaurepaire 2011). Le débat qui opposa, de Londres à l'Italie, Voltaire et Paolo Rolli est un témoin de cet espace littéraire, dans lequel les débats critiques circulent bien au-delà des frontières politiques et linguistiques. Si le français est la langue internationale de cette république, le passage d'une langue à l'autre joue un rôle important dans la diffusion large des modèles littéraires ${ }^{1}$. Après avoir retracé le parcours européen des réponses de Pao-

*jean-louis.haquette@univ-reims.fr

${ }^{1}$ Il convient de dire ici que, s'il fallait renvoyer à un cadre théorique, je préférerais à la notion de transfert culturel celle de polysystème. La première, insiste sur l'écart entre les cultures qui entrent en relation et les négociations et malentendus qui accompagnent le «transfert» (voir Espagne 2013). La seconde, dans l'époque qui nous occupe, est plus opératoire pour décrire et interpréter la circulation des idées dans un espace commun et sur un fond de valeurs partagées. Au-delà des variations locales, c'est dans un espace d'échanges, souvent nommé «république des lettres», que s’inscrit le débat entre Rolli et Voltaire. Son 
lo Rolli à l'Essai sur la poésie épique de Voltaire, j'essaierai de montrer comment se jouent, dans la rivalité des traductions du célèbre monologue d'Hamlet, des questions de poétiques autant que d'identités nationales. Il peut paraître paradoxal de centrer le propos sur un intermédiaire entre l'Angleterre et l'Italie, mais on verra que la France, incarnée en l'occurrence par Voltaire, joue un rôle central dans ce débat critique, et que les traductions en sont le vecteur principal.

\section{UN INTERMÉDIAIRE CULTUREL ACTIF}

Il convient d'abord de rappeler qui est Paolo Rolli (1687-1765). Même si les musicologues européens se souviennent de lui comme librettiste de plusieurs opéras de Haendel, il demeure dans un oubli relatif hors d'Italie. Moins connu que Giuseppe Baretti, il fut pourtant un important intermédiaire culturel entre la Grande-Bretagne et l'Italie dans l'Europe des Lumières. La partie la plus importante des activités littéraires de Rolli se déroula à Londres, où il vécut de 1715 à 1744 (cf. Dorris 1967, Sinopoli 2014, Bucchi 2003). Du point de vue italien, il fut le premier traducteur du Paradis perdu de Milton dans la langue de Dante; sa traduction n'est pas oubliée, puisqu'elle a connu deux rééditions au début de notre siècle (Rolli 2003, 2008).

Né à Rome, d'une famille originaire de Todi, Rolli fut élève de Giovanni Vincenzo Gravina, fondateur de l'Arcadia, défenseur comme on le sait d'une poésie «classique» et opposant des marinistes. Partisan d'une rénovation du passé littéraire glorieux de l'Italie et nourri des grands modèles (Dante, Pétrarque, Le Tasse), Rolli se fit remarquer par ses capacités d'improvisateur. Après avoir rencontré en Italie un aristocrate anglais, Thomas Herbert, comte de Pembroke, il se rendit à Londres; il y fréquenta le cercle littéraire de la duchesse de Shrewsbury, italienne de naissance, et celui de Lord Burlington, célèbre connoisseur, initiateur du néo-palladianisme en Angleterre. Il enseigna l'italien à la haute aristocratie londonienne, en particulier aux filles de George III, les princesses Caroline et Amélie, et au Prince de Galles, Frédéric. Il produisit des vers qui connurent un certain succès ${ }^{2}$ et procura de nombreux livrets d'opéra aux musiciens de son temps, Porpora, Bononcini, Haendel. Devenu membre de l'Académie royale de musique, il entretint plusieurs polémiques au sujet de la musique italienne (voir Dorris 1967: 192-204). Il eut aussi une activité régulière de traducteur, avec une large palette de langues: de l'italien à l'anglais (Boc-

caractère transnational, tout en incluant une forte présence de représentations nationales, est structurant. Je rejoins ici la position de Sinopoli (2014). Sur l'utilisation littéraire de la notion de polysystème, voir d'Hulst (1987) et la synthèse de Dimic (1993).

${ }^{2}$ On compte plus d'une dizaine d'éditions, en Angleterre et en Italie, de ses Rime. 
cace, Arioste), de l'anglais à l'italien (Steele, Milton), du latin à l'italien (Virgile, Overbecke), du grec à l'italien (Anacréon). La plus grande partie de ces traductions parut à Londres.

Du point de vue qui nous intéresse, il fut surtout, comme on l'a signalé, traducteur de Milton, dont la poésie l'avait visiblement saisi, alors même qu'elle était fort éloignée, par son appartenance générique comme par son style, de ses productions versifiées ainsi que des modèles qui lui étaient naturels. Cette admiration s'ajouta à son attachement au Tasse pour le conduire à croiser le fer avec l'auteur de la Henriade, en publiant en 1728, en anglais, Remarks upon Mr. Voltaire's Essay on the Epic Poetry of the European Nations (Rolli 1728a) ${ }^{3}$.

\section{L'AUTORITÉ DÉFIÉE: ROLLI \& L'ESSAI SUR LA POÉSIE ÉPIQUE}

Dans le domaine du débat littéraire, la contribution de Paolo Rolli, même si elle a été éclipsée par la célébrité de Voltaire, n'est pas négligeable. D'après Silvio Fassini (1907) et Simone Carpentari-Messina (1978), Voltaire, qui ne consacre à Rolli qu'une très brève mention négative ${ }^{4}$, aurait tenu compte de ses remarques dans la version français de l'Essai sur la poésie épique de 1733. L'intérêt du débat n'est cependant uniquement là. Il réside dans la conception de la littérature, et non seulement de la littérature italienne ou anglaise, qui pousse Rolli à s'opposer à l'autorité déjà réelle de l'auteur d'Edipe puis de la Henriade. J'insisterai ici sur l'histoire éditoriale de ses Remarks, qui est révélatrice des enjeux liés à cette polémique. Même si l'on peut considérer qu'avoir pour «ennemi» Voltaire pouvait entrer dans une stratégie auctoriale, il me semble bien que d'autres motivations sont à l'œuvre. On peut rappeler, par ailleurs, que c'est 26 ans après la publication de l'essai voltairien que Baretti (1753) lancera en anglais sa propre réponse, intitulée $A$ Dissertation upon the Italian Poetry in which are interspersed some Remarks on Mr. Voltaite's Essay on the Epic Poets.

Pour mieux comprendre la portée critique que les Remarks possèdent dans le parcours de Rolli, il faut commencer par retracer l'histoire éditoriale, bien connue mais compliquée, de l'Essai sur la poésie épique de Voltaire,

${ }^{3}$ Le débat avec Voltaire a été déjà présenté et analysé par Fassini (1907) et Carpentari-Messina (1978).

${ }^{4}$ «[... $]$ quiconque écrit en vers doit écrire en beaux vers, ou ne sera point lu. Les poètes ne réussissent que par les beautés de détail. Sans cela Virgile et Chapelain, Racine et Campistron, Milton et Ogilby, le Tasse et Rolli, seraient égaux.» Lettre à Jacob Vernet, 14 septembre 1733 (Best D 653), citée dans Carpentari-Messina (1978 : 103, n. 15). 
pour la croiser avec le parcours de la réponse qu'il suscite chez l'auteur italien.

Voltaire, âgé de 32 ans, avait été forcé de s'exiler à Londres à cause de son affaire avec le chevalier de Rohan ${ }^{5}$. Il avait emporté dans ses bagages le manuscrit de la Henriade, œuvre qui devait confirmer son statut littéraire de grand écrivain, suite au succès déjà obtenu avec Edipe en $1718^{6}$. Afin de préparer l'opinion à l'édition londonienne du texte, mais surtout d'accompagner la souscription qui avait été lancée pour financer la publication de l'épopée, Voltaire écrit, en anglais, et publie en 1727, An Essay on the Epic Poetry of European Nations. From Homer down to Milton (Voltaire $1727)^{7}$. Le texte est une manière de préface à son œuvre épique. Il parcourt en neuf chapitres le chemin qui mène à sa propre épopée, partant bien sûr d'Homère, dont il fait un éloge mitigé, pour arriver à Milton, en passant par Virgile, Lucain, Trissin, Camoens, Le Tasse et Ercilla ${ }^{8}$.

C'est dès le début de l'année suivante, que Paolo Rolli publie ses Remarks upon Mr. Voltaire's Essay on the Epic Poetry of the European Nations. L'ouvrage est composé comme une série de notes au texte de Voltaire. La disposition typographique est celle que l'on trouve à la fin des ouvrages poétiques de l'époque: reprise d'un passage du texte source et commentaire. Après une introduction de 5 pages, trente-neuf passages sont commentés, portant essentiellement sur Homère, sur le Tasse, et sur Milton (toutes les pages consacrées au poète anglais sont annotées). L'avertissement insiste sur Le Tasse et sur Milton, soit les poètes épiques modernes qui représentent respectivement sa patrie et de son pays d'adoption. Il faut insister sur cette double défense: Rolli ne s'enflamme pas uniquement en faveur de la littérature italienne. Certes il considère que Voltaire n'a qu'une connaissance de seconde main de la littérature italienne et profère des accusations injustifiées contre Le Tasse et plus généralement contre la poésie italienne, qui serait affligée d'un goût pour le faux brillant. Mais il défend aussi la sublimité de Milton, qui excède le cadre rationnalisant de la poétique épique

${ }^{5}$ L'histoire en a retenu la réplique voltairienne: «Je commence mon nom, vous finissez le vôtre» (voir Pomeau 1985).

${ }^{6} \mathrm{Si}$ le succès fut littéraire, il fut aussi lié au scandale des amours incestueuses supposées par l'opinion publique entre le Régent et sa fille ainée, la duchesse de Berry.

${ }^{7}$ L'essai est publié à la suite de An Essay upon the Civil Wars of France. Extracted from Curious Manuscripts. Cet essai historique est aussi lié à la Henriade, qui célèbre l'action pacificatrice d'Henri IV. Le texte, à cause de la censure, fut publié en Hollande en 1729 dans la traduction de l'abbé Granet.

${ }^{8}$ La présence de Trissin (L'Italie libérée des Goths, 1547) et d'Ercilla (La Auraucana, 1589 ) est inattendue et montre l'intérêt de Voltaire pour les tentatives de renouvellement du genre épique. 
voltairienne ${ }^{9}$. En se faisant l'avocat de deux auteurs qu'il admire, face à des accusations jugées injustes, c'est aussi une certaine idée de la poésie qu'il défend. Elle privilégie l'effet sur le lecteur au respect des codes poétiques, l'énergie des images aux bienséances, le sublime au beau ${ }^{10}$.

C'est aussi en 1728 que Voltaire fait paraître la Henriade. En mai de la même année, sans l'aveu de Voltaire, l'abbé Desfontaines (1685-1745) publie sa traduction de l'Essai. Dès le mois de juillet, soit avec un temps de réaction très bref, paraît la traduction française des remarques de Rolli (1728b), par l'abbé Antonini ${ }^{11}$. La «Préface du traducteur» est claire: il espère arrêter en France «les idées désavantageuses que M. de Voltaire y pourrait inspirer de nos Auteurs, comme M. Rolli les a prévenues en Angleterre» (Rolli 1728b : n.p. [ii]).

Il est vraisemblable que Rolli soit intervenu dans la publication de la traduction. Il était sans doute désireux d'accompagner le parcours du texte voltairien, jugeant utile ne pas le laisser sans contestation en France non plus. Comme il n'écrivait pas le français, il s'adressa à un traducteur. On ne sait quels furent les liens entre l'auteur et son traducteur. Celui-ci, dans la préface, se réclame clairement de l'anti-marinisme, dans la lignée de Gravina. Il est possible que l'abbé Antonio Conti, longtemps établi à Paris, et que Rolli connaissait, ait servi d'intermédiaire ${ }^{12}$.

Mais l'histoire ne s'arrête pas là. En effet, parallèlement à son combat avec Voltaire, Rolli avait entrepris la traduction en italien du Paradis perdu de Milton (ce qui justifie en partie l'insistance sur ce poème dans les $R e$ marks $)^{13}$. Les six premiers livres paraissent à Londres en 1729 (Rolli 1729), la même année que la première traduction française de Dupré de Saint-Maur

${ }^{9}$ La position de Rolli s'appuie en partie sur les célèbres analyses d'Addison dans sa série d'articles du Spectator en 1712 (voir Forlesi 2017).

${ }^{10}$ Il n'est pas possible ici d'entrer dans un examen détaillé de ces idées, qui mériteraient d'être replacées dans le cadre de l'évolution des paradigmes poétiques dans l'Europe des Lumières. On se contentera d'en indiquer, chemin faisant, quelques caractéristiques.

${ }^{11}$ Après des études à Naples, Annibale Antonini (1702-1755) fut pendant 25 ans maître de langue italienne à Paris. Il est l'auteur d'une grammaire italienne et d'un dictionnaire italien, latin, français qui eurent un certain succès. Il eut aussi une activité de traducteur et d'éditeur de textes italiens à Paris (voir Dizionario bibliografico degli italiani 1961).

${ }^{12}$ Rolli (1730: 282) évoque une conversation autour de Milton chez l'abbé Conti dans ses Osservazioni. L'abbé Antonio Conti (1677-1749), oratorien, séjourna à Londres (1715-1718) puis à Paris jusqu'en 1726; il fut un proche du comte de Caylus et de sa mère. Sur son influence littéraire, voir Hamm (1956) et Robertson (1923).

${ }^{13}$ Fassini (1907: 88, n. 5) a pu montrer que Rolli avait commencé cette traduction dès juin 1717, soit deux ans après son arrivée à Londres. Sur le travail de de Rolli sur la traduction de Milton, voir Bucchi (2006). 
à Paris ${ }^{14}$. L'année suivante, en 1730, la traduction des six premiers livres est imprimée en Italie, à Vérone, chez Tumermani, et dédiée à Maffei. Rolli l'accompagne cette fois d'une version italienne des Remarks avec le titre suivant: Osservazioni sopra il libro del signor Voltaire che esamina l'epica poetica delle nazioni europee scritto originalmente in Inglese e in Londra stampate nel 1728 poi nella propria lingua tradotte.

Dans une lettre de 1737, Rolli expliquera cette association, qui n'est pas justifiée uniquement par le fait que Milton fasse l'objet d'une partie des remarques:

In fronte alla ristampa fatta dal Tumermani in Verona della mia traduzione de' sei primi libri Miltoniani come primo saggio dell'opra or compiuta, si ristampò quella mia risposta da me tradotta in nostra lingua ed iscritta al mio vecchio amico marchese Scipione Maffei. V. E. [...] vedrà con quale acre inchiostro gl'Italiani, che non son mai gli aggressori, san vendicarsi di chi gli offende. Sono però i Francesi scusabili per tener sempre contra noi l'arco teso, e a dritto e a torto ferirci: siamo i soli che con miglior armi, del pari che con miglior senno, ogni volta che insolentemente n'escono, gli ripulsiamo nei lor angusti confini (citée dans Fassini 1907: 99) ${ }^{15}$.

Le vocabulaire est terriblement belliqueux et Rolli file la métaphore guerrière, qui semble presque renvoyer aux guerres d'Italie du XVIe siècle... Il est donc bien question de la littérature nationale italienne, et de sa défense face à l'ennemi français, mais la dédicace à Maffei indique aussi un manifeste en faveur d'une certaine idée de la littérature.

Lorsqu'en 1733, Voltaire, ayant réécrit l'Essai sur la poésie épique en français (et l'ayant modifié en partie) le fait paraître, Rolli ne réagit pas par une réédition des Remarques françaises. Il savoure peut-être les modifications concernant la littérature italienne comme une prise en compte implicite des critiques. C'est, on l'a vu, l'opinion de Fassini et Carpentari-Messina. Indépendamment de la polémique londonienne, puis parisienne, Rolli va cependant persister à continuer de joindre à sa traduction de Milton les Remarks, devenues Osservazioni ${ }^{16}$. Dans d'édition in-12 de 1740, toujours chez Tumermani à Vérone, mais avec une fausse adresse de Paris, le second

${ }^{14}$ Le fait mérite d'être souligné: dans la réception continentale du Paradis perdu, la France ne sert pas de relai vers l'Italie, et la diffusion se fait simultanément dans les deux pays, grâce à Paolo Rolli. Il faut signaler qu'en France c'est l'abbé Antonio Conti qui fut l'un des premiers à s'intéresser au poème de Milton (voir Gillet 1975).

${ }^{15}$ Lettre à Domenico Passionei (nonce apostolique à Vienne), Londres, 23 avril 1727.

${ }^{16}$ Il faut préciser que l'édition complète de la traduction du Paradis perdu (Londres, Charles Bennet, 1735) ne les inclut pas. Comme elles ont paru en anglais à Londres, Rolli ne les ajoute pas; la traduction italienne des Osservazioni est donc bien destinée au public italien (alors que la traduction du poème de Milton était d'abord destinée aux Anglais italophiles). 
volume est occupé par une vie de Milton, les essais d'Addison, traduits en italiens, et les Osservazioni. Ces dernières occupent 100 pages sur 267. Alors qu'elles étaient en tête du volume, en 1730, elles sont situées à la fin, et font partie de l'apparat critique qui accompagne le texte, au même titre que les essais d'Addison. En 1742, dans la grande édition in-folio, toujours chez Tumermani, elles sont toujours présentes, en fin de volume ${ }^{17}$. Malgré leur position finale, on les entrevoit, dans les deux éditions, dès la page de titre sous la désignation large: «Aggiunte alcune osservazioni critiche».

De cette histoire éditoriale qui s'étend sur 12 ans $^{18}$, on ne peut que conclure que Rolli accordait une importance toute particulière à sa réponse à Voltaire: il s'agissait de défier son autorité pour rétablir ce qu'il estimait une vision juste de la littérature italienne comme du chef d'œuvre dont il s'était fait le héraut italien. Mais ces remarques, qui n'étaient pas faites d'abord pour être lues de façon indépendante, gagnèrent finalement leur autonomie. Elles remplacent en quelque sorte l'art poétique que Rolli n'a jamais écrit. Elles réunissent Le Tasse et Milton, malgré leurs différences, comme modèles poétiques ${ }^{19}$. Il s'agit aussi du texte le plus international de Paolo Rolli, puisqu'on en compte trois versions, en anglais, en français, et finalement en italien.

\section{POÉTIQUE DE LA TRADUCTION: REGARDS CONTRASTÉS SUR LE MONOLOGUE «TO BE OR NOT TO BE»}

Le défi à Voltaire se prolonge, de façon plus discrète, à propos de la traduction du monologue «Être ou ne pas être» d'Hamlet. On se souvient que Voltaire en avait donné une traduction dans la version anglaise des Lettres philosophiques, en 1733 (Voltaire 1733) ${ }^{20}$, puis dans la version française, l'année suivante (Voltaire 1734) ${ }^{21}$. Paolo Rolli publie à Londres, en 1739,

${ }^{17}$ Elles y occupent les pages 71-96.

${ }^{18}$ Et même au-delà, car les Osservazioni sont toujours présentes la réédition de 1758 (Paris [Venise], Bartolomeo Occhi).

${ }^{19}$ Rolli répond longuement aux critiques de Voltaire au sujet de ces deux auteurs, en défendant notamment la liberté d'invention, contre une approche 'rationalisante'. Les 'fictions' et les 'épisodes' mis en cause par le critique français sont justifiés en grande partie par l'effet sur le lecteur et au nom de l'imagination poétique. La défense du célèbre «darkness visible» de Milton est notamment caractéristique de cette approche (Rolli 1740: 288-292). Rolli y défend la liberté du grand poète d'oser («aventurarsi») par opposition à l'historien qui ne doit pas se risquer aux envols poétiques («poetici voli»).

${ }^{20}$ Lettre XVIII. Le texte anglais et la traduction étaient donnés tous les deux, ce qui ne laisse pas de surprendre dans un texte destiné au public anglais.

${ }^{21}$ Seule la «traduction» est donnée. 
à la suite de la traduction des Odes d'Anacréon (Rolli 1939: 97-99), sa propre version, en italien, qui est la première dans cette langue. On retrouve ici la centralité du relais français entre l'Angleterre et l'Italie, et la position cardinale acquise par Voltaire dans le champ littéraire ${ }^{22}$.

L'auteur de la Henriade avait introduit ce qui est en fait une imitation partielle du célèbre monologue, en rappelant la supériorité de l'original et en utilisant l'opposition topique entre peinture et gravure: «J'ai hasardé de traduire quelques morceaux des meilleurs poètes anglais: en voici un de Shakespeare. Faites grâce à la copie en faveur de l'original; et souvenezvous toujours, quand vous voyez une traduction, que vous ne voyez qu'une faible estampe d'un beau tableau» (Voltaire 1879a: 149).

Il défend aussi sa conception de la traduction, qui privilégie l'idée sur la forme: «Ne croyez pas que j'aie rendu ici l'anglais mot pour mot; malheur aux faiseurs de traductions littérales, qui en traduisant chaque parole énervent le sens! C'est bien là qu'on peut dire que la lettre tue, et que l'esprit vivifie» (Voltaire 1879a: 149).

Le recours à l'anathème et à l'image biblique donne une véhémence certaine à la formulation de ce qui est la position dominante à l'époque. Paolo Rolli ne la partage pas du tout: pour lui traduire doit donner la priorité au texte de départ sur l'adaptation à la poétique ou au goût du public visé; car, si Voltaire affirme privilégier le sens, c'est qu'il juge la poétique shakespearienne inacceptable par le goût de la «nation française». Sans s'étendre sur ses motivations, Rolli indique brièvement ce qui l'a conduit à traduire le monologue en italien:

Monsieur de Voltaire, in una delle sue Lettere sovra la nazione britannica, ragionando del famoso tragico Shakespeare, per darne qualche saggio tradusse il soliloquio nella tragedia d'Hamleto principe di Danimarca. Questa litteral traduzzione mostrerà quant'egli deviò da' sentimenti e dallo stile di quell'originalmente sublime Poeta. I Versi originali sono 32, tradotti 29 (Rolli 1939: 96).

De façon assez inattendue, cette traduction est comme une apostille aux Remarks: il s'agit toujours de contredire Voltaire, et de montrer, comme à propos de Milton, qu'il ne rend pas justice au sublime de cette poésie, dont ni le style, ni les idées ne sont en fait respectés. Voilà comment naquit la première traduction en italien du monologue «To be or not to be»: pour montrer aux Anglais qui lisaient l'italien ${ }^{23}$ que leur grand dramaturge avait

${ }^{22}$ Le fait que ses idées soient critiquées ne remet pas en cause cette position; les polémiques contribuent même d'une certaine façon à la confirmer.

${ }^{23}$ Il faut bien être conscient, dans ces débats, des destinataires visés, dont le système de référence n'est pas absent de l'esprit des auteurs. L'abbé Antonini accuse d'ailleurs Voltaire d'avoir voulu «jeter de la poudre aux yeux» des Anglais en faisant quelques éloges 
été trahi par l'auteur de la Henriade. C'est donc bien toujours l'autorité de Voltaire qu'il s'agit de défier, mais c'est aussi une poétique, moderne, de la traduction qui s'affirme. La conception rollienne est en effet à bien des égards encore la nôtre; elle est résolument tournée vers le texte dont elle veut s'efforcer de faire sentir les beautés, et non plus vers le public auquel il faudrait adapter l'auteur. Cette conception avait d'ailleurs déjà été explicitée dans la «Vita di Milton», qui accompagne la publication de la traduction des six premiers chants du Paradis perdu, à Londres en 1729. Il convient de citer un peu longuement le texte, car s'il peut apparaître comme un plaidoyer pro domo sua ${ }^{24}$, il possède aussi une dimension de manifeste de traduction:

Di questa mia traduzzione io penso ch'ella sia la più esatta metafrasi che siasi mai letta, e ciò per l'estrema correlazione delle sintassi nelle due lingue e particolarmente nello stil miltoniano; e siccome io pretendo d'aver non solo litteralmente tradotto i sensi di Milton, ma pur anche la poesia; così dico non esser nell'opera mia parte alcuna ch'io voglia scusare come deficiente di sublimità e poetica bellezza per aver voluto esser traduttor litterale. No, non basta per ben tradurre tali opere spiegarne il senso in altra lingua. Tutte le più trasportatrici bellezze che in delicati e talor minutissimi tratti scintillano, tutte allora si perdono, poichè lo scheletro solo, e non il bellissimo corpo nelle sue intere fattezze e negli ornamenti della vaghissima veste, allor se ne mostra (Rolli 1730: 157)

La position de Rolli va à l'encontre de la doxa de son époque, la traduction littérale des poètes, surtout modernes, étant généralement dévalorisée. Ici, sur une prémisse contestable (la proximité syntaxique de l'anglais et de l'italien) c'est une défense et illustration de la littéralité de la traduction que se livre Rolli. Elle le conduit à utiliser un hellénisme, metafrasi, pour souligner son attachement à la lettre du texte ${ }^{26}$. Pour notre poète, traduire littéralement est la condition nécessaire de la préservation des beautés

de Milton pour mieux diffamer ensuite le Paradis perdu. Cf. la «Préface du traducteur» dans Rolli (1728a: n. p. [xi]).

${ }^{24}$ Le passage cité suit la mention de la parution en France de la traduction de Dupré de Saint-Maur, dont Rolli regrette qu'il n'ait pas mieux compris l'original. Il affirme que sa traduction en fera voir les erreurs et les manques.

${ }^{25}$ Comme la «Vita di Milton» n'est pas paginée dans l'édition londonienne de 1729 , je la cite dans l'édition véronaise Tumermani de 1730. La page de titre de cette édition n'annonce pas la vie de Milton (Rolli 1730: 115-164) qui suit les Osservazioni. Celles-ci, en revanche, sont mentionnées sur la page de titre. L'ordre des pièces est inhabituel, car c'est généralement la vie de l'auteur qui est en tête de volume, après les pièces dédicatoires. Cela montre bien l'importance qu'accorde Rolli à ses remarques, qui peuvent être vues comme une introduction à Milton.

${ }^{26}$ Le mot est absent des éditions du Vocabolario degli Accademici della Crusca. En revanche il est attesté, à partir du français «métaphrase», dans Roujoux (1826: 309). 
poétiques de l'original. Il ne suffit donc pas de rendre le sens, mais les beautés de la forme, ce qui exige une fidélité stylistique. Le changement de paradigme s'exprime dans le choix d'un renouvellement des métaphores et d'une valorisation inversée liée à l'acte de traduction. L'image de l'estampe, inférieure au tableau, est remplacée par celle du squelette et de la forme extérieure du corps: pour Rolli la traduction n'est inférieure à l'original (comme l'estampe au tableau) que si elle sacrifie la forme. Elle aboutit alors à la réduction de la poésie à son squelette. La vraie traduction consiste à «transporter» les beautés d'une langue à une autre. Pour contestable que soit le point de départ, qui fait bon marché de la différence des structures des langues, l'insistance sur la matérialité langagière du style poétique est remarquable. La volonté de fidélité se traduit aussi dans une disposition typographique très particulière: Rolli adopte dès l'édition londonienne de 1730 une double numérotation des vers: de la traduction à gauche, de l'original en bout de ligne à droite.

On notera enfin que, comme dans le cas de Shakespeare, le sublime est la notion alléguée par Rolli pour rendre compte du génie poétique. Ici aussi c'est d'un changement de paradigme qu'il s'agit: c'est bien le sublime qui rendra acceptable, même aux yeux d'un Voltaire, certains aspects de la poétique miltonienne fort éloignés des normes classiques ou néo-classiques. La poétique du beau commence à céder la place à l'esthétique du sublime, ce qui est un basculement majeur dans la réflexion critique européenne ${ }^{27}$.

Cette vision de Shakespeare s'exprimait déjà dans les Osservazioni, où le dramaturge anglais était loué pour l'énergie de son théâtre, au-delà de la question des bienséances qui occupera la critique française tout au long du siècle, et Voltaire en premier lieu: «Quel che farà sempre scintillare la gloria dell'inglese tragico Shakespar [sic!] sul teatro Britanno, è quella forza d'evidenza nel dipingere i caratteri degl'Inglesi et de' Romani grand'uomini nelle sue tragedie; sì vivamente rappresentandoli nelle loro virtù, temperamenti e difetti» (Rolli 1740: 297).

Cette «force d'évidence» est liée au refus de l'idéalisation, à une proximité avec l'humanité saisie dans sa vérité. Il ne s'agit pas ici, puisqu'on est au théâtre, d'une simple reprise de la tradition rhétorique de l'hypotypose, mais d'une inflexion esthétique, qui relativise la question des bienséances. Reprenant la vieille question de savoir si, au théâtre, il faut plutôt représenter

Il est bien sûr un synonyme savant de traduction, mais témoigne de l'insistance de Rolli sur la littéralité.

${ }^{27}$ Une étude détaillée de la conception du sublime dans la pensée critique de Rolli permettrait de montrer dans quelle mesure l'auteur, tout en restant tributaire de Longin et de Boileau, s'inscrit dans un sublime de l'origine primitive, qui comporte une sombre grandeur, dont Burke sera le théoricien. 
les hommes tels qu'ils sont ou tels qu'ils devraient être, Rolli répond sans ambigüité:

Gli uomini possono esser rappresentati dell'arte, o com'eglino sono, o com'esser dovrebbono; ma il descriverli come sono, è a mio senso assai più difficile, non che più dilettevol più utile, perché gli spettatori gli ritrovano più rassomiglianti a se stessi; onde il verisimile acquista allora tutte le forze del vero per far più viva quelle impressione nello spettatore, la quale è il gran fine dell'opra, e la maggior gloria del poeta (Rolli 1740: 297).

Ici encore les règles sont subordonnées au plaisir du spectateur, qui est la pierre de touche du théâtre. Rolli prend le même parti que les partisans du coloris en peinture, tels De Piles quelques années plus tôt, celui de la force de la représentation, qui repose sur les couleurs de la réalité. Cette poétique de la vive peinture, qui a eu longue hérédité européenne (voir Hénin 2003), est répétée à plusieurs reprises dans les Osservazioni. Elle y prend un tour nouveau au moins par sa formulation, car il s'agit de donner corps à l'invisible: «Occupare gli occhi di chi ascolta o legge, del pari che la mente, dando corpo e visione a cose che non gli hanno, è l'incanto della Poesia» (Rolli 1740: 357).

Les questions de traduction ne sont donc pas affaire de linguiste seulement, mais sont liées à un ensemble de convictions, qui font de Rolli un acteur intéressant du renouvellement des paradigmes littéraires de son temps, entre Angleterre, France et Italie.

C'est sur une note plus paradoxale que j'ai choisi de clore ce parcours. Voltaire lui-même, bien qu'horrifié par la vogue grandissante de Shakespeare sur le continent européen, exprime parfois de façon inattendue sa fascination face à Shakespeare, et ce au moment même où il prend les armes contre le «sauvage ivre» qui menace le système dramatique français. Il se rapproche de façon inattendue de Rolli, car c'est la traduction littérale qui se fait la révélatrice de la vraie grandeur de la poésie anglaise comme du barde britannique. Dans son Appel à toutes la nations d'Europe (1761), réponse à un article du Journal encyclopédique qui osait préférer Shakespeare à Corneille, Voltaire donne en effet la traduction littérale du monologue «To be or not to be». Cette traduction doit en révéler la vulgarité, dont la version des Lettres philosophiques avait gommé les aspérités. Pourtant, alors même que Voltaire s'attarde à «démolir» le texte célèbre, il concède une réelle grandeur non seulement à la langue anglaise, mais aussi au monologue lui-même, qui relève finalement d'un certain sublime, celui d'une beauté irrégulière mais puissante. Sans remettre en cause le credo «classique» de l'auteur de la Henriade, cette concession à la possibilité d'un autre goût, dont Homère, La Bible, Milton et Shakespeare peuvent être les représentants, contient en germe une autre esthétique, qui s'affirma au long du siècle: 
À travers les obscurités de cette traduction scrupuleuse, qui ne peut rendre le mot propre anglais par le mot propre français, on découvre pourtant très-aisément le génie de la langue anglaise; son naturel, qui ne craint pas les idées les plus basses, ni les plus gigantesques; son énergie, que d'autres nations croiraient dureté; ses hardiesses, que des esprits peu accoutumés aux tours étrangers prendraient pour du galimatias. Mais sous ces voiles on découvrira de la vérité, de la profondeur, et je ne sais quoi qui attache, et qui remue beaucoup plus que ne ferait l'élégance; aussi il n'y a presque personne en Angleterre qui ne sache ce monologue par cœur. C'est un diamant brut qui a des taches: si on le polissait, il perdrait de son poids (Voltaire 1879b: 203).

\section{ANNEXE: HAMLET, III, 1}

Shakespeare (1995: 204 et 206)

To be, or not to be, that is the question Whether 'tis nobler in the mind to suffer The slings and arrows of outrageous fortune, Or to take arms against a sea of troubles, And by opposing end them: to die, to sleep No more; and by a sleep, to say we end The heart-ache, and the thousand natural shocks That flesh is heir to - 'tis a consummation Devoutly to be wished. To die, to sleep To sleep, perchance to dream - aye, there's the rub, For in that sleep of death, what dreams may come, When we have shuffled off this mortal coil, Must give us pause. There's the respect That makes calamity of so long life:

For who would bear the whips and scorns of time, The oppressor's wrong, the proud man's contumely, The pangs of despised love, the law's delay, The insolence of office, and the spurns That patient merit of the unworthy takes, When he himself might his quietus make With a bare bodkin? Who would fardels bear, To grunt and sweat under a weary life, But that the dread of something after death, The undiscovered country, from whose bourn No traveller returns, puzzles the will, And makes us rather bear those ills we have, Than fly to others that we know not of. Thus conscience does make cowards of us all, 
And thus the native hue of Resolution

Is sicklied o'er, with the pale cast of thought, And enterprises of great pitch and moment, With this regard their currents turn awry, And lose the name of action.

\section{Voltaire (1879a: 149)}

Demeure; il faut choisir, et passer à l'instant

De la vie à la mort, ou de l'être au néant.

Dieux cruels! s'il en est, éclairez mon courage.

Faut-il vieillir courbé sous la main qui m’outrage,

Supporter ou finir mon malheur et mon sort?

Qui suis-je? Qui m'arrête? Et qu'est-ce que la mort?

C'est la fin de nos maux, c'est mon unique asile;

Après de longs transports, c'est un sommeil tranquille;

On s'endort, et tout meurt. Mais un affreux réveil

Doit succéder peut-être aux douceurs du sommeil.

On nous menace, on dit que cette courte vie

De tourments éternels est aussitôt suivie.

O mort! Moment fatal! Affreuse éternité!

Tout cœur à ton seul nom se glace, épouvanté.

Eh! qui pourrait sans toi supporter cette vie,

De nos Prêtres menteurs bénir l'hypocrisie,

D'une indigne maîtresse encenser les erreurs,

Ramper sous un Ministre, adorer ses hauteurs, Et montrer les langueurs de son âme abattue

À des amis ingrats qui détournent la vue?

La mort serait trop douce en ces extrémités;

Mais le scrupule parle, et nous crie: «Arrêtez.

Il défend à nos mains cet heureux homicide,

Et d'un Héros guerrier fait un chrétien timide.

$$
\text { Rolli (1739: 97-99) }
$$

Essere o no, la gran Questione è questa:

Qual nella Mente è forte più? Soffrire

Colpi e Saette d'oltraggiosa Sorte;

O prender l'Armi contra un mar d'Affanni, E dar loro, in opporsi, a un tratto il fine?

Morir! Dormire: Altro non è. Nel Sonno,

Dicon, che fine avrà il Cordoglio, e mille,

Retaggio della Carne, altre Sciagure: 
Consumazion, d'avida Brame oggetto!

Morir! Dormir! Dormir? forse Sognar! Ah

Qui è l'Intoppo! Chè in quel Sonno di Morte

Quai Sogni possan venir poi che avremo

Scossa alla fin questa mortale Spoglia:

Sospendon l'Alma. Ecco il Ristesso ond'anno

Nostre Calamità si lunga Vita.

Altrimente, Chi mai soffrir le atroci

Del suo tempo vorrai Sferzate e Scherni,

Torri d'Oppressione, Onte d'Orgoglio,

Fiere Agonie di disprezzato Amore,

Leggi indugiate, Autorità insolente,

E quei che il Merto paziente oppresso

Aspri riceve dal Demerto Oltraggi;

Quando ci dar si potesse alta Quiete

Con la punta d'un Ago? E chi la grave

Soma portar vorria; Chi sotto a stanca

Vita, gemer, sudar; senza il Terrore

Di spaventevol Cosa appo la Morte?

Quelle Contrade incognite dal cui

Confine mai Viaggiator non torna,

La Volontà sgomentano, e ci fanno

Piuttosto i Mali sostener presenti;

Che sciorre ad altri sconosciuti il volo.

Coscienza Cosi di tutti Noi

Tanti Codardi fa; così 'l nativo

Suo robusto Color Risoluzione

Smarrisce in pensierosa Pallidezza:

E le Imprese di grande Auge e Momento

Arrestate da un tal Riguardo; svolgono

Lor Corrente, e d'Azzion perdono il Nome.

\section{BIBLIOGRAPHIE}

Baretti, G. (1753). A Dissertation upon the italian poetry in which are interspersed some remarks on Mr. Voltaire's Essay on the epic poets. London: R. Dodsley.

Beaurepaire, P.-Y. (2011). L'Europe au siècle des Lumières. Paris: Ellipses. Bucchi, G. (2003). L'italiano in Londra: Paolo Rolli editore dei classici italiani. Versants, 43, 229-265.

Bucchi, G. (2006). Un esemplare del Paradiso perduto postillato da Paolo Rolli. Seicento \& Settecento, 1, 55-76.

Carpentari-Messina, S. (1978). Voltaire et Paolo Rolli: les deux versions de l'Essai sur la poésie épique. In L. Desvignes (éd.), Travaux compara- 
tistes (p. 81-110). Saint-Etienne: Centre d'études comparatistes et de recherche sur l'expression dramatique.

D’Hulst, L. (1987). L'Evolution de la poésie en France (1780-1830). Introduction à une analyse des interférences systémiques. Louvain: Leuven University Press.

Dimic, M. V. (1993). Polysystem Theory. In I. Makaryk (éd.), Encyclopedia of Contemporary Literary Theory (p. 151-155). Toronto: University of Toronto Press.

Dizionario bibliografico degli italiani (vol. 3). (1961). Roma: Istituto dell'Enciclopedia Italiana.

Dorris, G. E. (1967). Paolo Rolli and the italian circle in London, 1715 1744. La Haye: Mouton.

Espagne, M. (2013). La notion de transfert culturel. Revue Sciences/Lettres, 1 . Texte disponible en ligne à la page http://journals.openedition. org/rsl/219, consulté le 30/01/2019.

Fassini, S. (1907). Paolo Rolli contro il Voltaire. Giornale Storico della letteratura italiana, XXXIX, 83-98.

Forlesi, S. (2017). Una polemica «mediata» tra Addison e Voltaire intorno al genere epico. Lo Spectator nel cantiere del Paradiso perduto di Paolo Rolli. In G. Bucchi \& C. E. Roggia (éds.), La critica letteraria nell'Italia del Settecento. Forme e problemi (p. 81-91). Ravenna : Longo.

Gillet, J. (1975). Le Paradis perdu dans la littérature française de Voltaire à Chateaubriand. Paris: Klincksieck.

Hamm, V. (1956). Antonio Conti and English aesthetics. Comparative Literature, VIII, 12-27.

Hénin, E. (2003). Ut pictura theatrum. Théâtre et peinture de la Renaissance italienne au classicisme français. Genève: Droz.

Pomeau, R. (1985). Voltaire en son temps. Vol. 1. D'Arouet à Voltaire. Oxford: Voltaire Foundation / Paris: J. Touzot.

Robertson, G. (1923). Studies in the genesis of romantic theory in the eighteenth century. Cambridge: Cambridge University Press.

Rolli, P. (1728a). Remarks upon Mr. Voltaire's Essay on the epick poetry of the european nations. London: T. Edlin.

Rolli, P. (1728b). Examen de l'Essai de M. de Voltaire sur la poésie épique par M. Paul Rolli. Traduit de l'Anglais, par M. L. A**. Paris: Rollin fils.

Rolli, P. (1729). Del paradiso perduto poema inglese di Giovanni Milton, libri sei, parte prima, tradotti da Paolo Rolli, compagno della Reale Società in Londra, l'acclamato nell' Accademia degli 'intronati in Siena, accademico quirino e pastore arcade in Roma. London: Samuel Aris. 
Rolli, P. (1730). Il Paradiso perduto di John Milton. Verona: Tumermani.

Rolli, P. (1740). Il Paradiso perduto di John Milton. Paris [Verona]: Tumermani.

Rolli, P. (1939). Delle odi di Anacreonte. London: s.e.

Rolli, P. (2003). Il Paradiso perduto di John Milton, éd. F. Longoni. Roma: Salerno «I Diamanti».

Rolli, P. (2008). Il Paradiso perduto di John Milton, éd. L. Alcini, Roma: Aracne.

Roujoux, P. G. (1826). Dizionario classico francese italiano (vol. 2). Paris: Librairie classique élémentaire.

Shakespeare, W. (1995). Hamlet, édition bilingue. Paris: Flammarion GF. Sinopoli, F. (2014). Dalla repubblica letteraria alla letteratura europea: Paolo Rolli tra Italia e Inghilterra. In B. Alfonzetti et al. (éds.), I cantieri dell'italianistica. Ricerca, didattica e organizzazione agli inizi del XXI secolo. Roma: Adi Editore. Texte disponible en ligne à la page http://www.italianisti.it/Atti-di-Congresso? $\mathrm{pg}=\mathrm{cms} \& \mathrm{ext}=\mathrm{p} \& \mathrm{cms}$ codsec $=14 \& \mathrm{cms}$ codcms $=581$, consulté le 30/01/2019.

Voltaire (1727). An Essay on the Epic Poetry of European Nations. From Homer to Milton. London: Samuel Jallason.

Voltaire (1733). Letters concerning the english nation. London: C. Davis, A. Lyon.

Voltaire (1734). Lettres écrites de Londres sur les Anglais et autres sujets. Rouen: Jorre.

Voltaire (1879a). Lettres philosophiques. In Voltaire, Euvres complètes (Vol. 22, pp. 75-188). Paris: Garnier.

Voltaire (1879b). Appel à toutes les nations d'Europe. In Voltaire, Euvres complètes (Vol. 24, pp. 191-222). Paris : Garnier.

\section{PAOLO ROLLI \& VOLTAIRE: LITERARY MODELS AND CRITICAL CONTESTATION}

\section{Summary}

The critical debate between Voltaire and Paolo Rolli is an example of the fruitful transnational exchange and the diffusion of literary models in $18^{\text {th }}$ century Europe; translations play a central role in this circulation of values. The complex editorial history of texts and comments written and translated into English, French and Italian brings out opposing views on epic poetry and, more generally, on literature. On the other hand, Voltaire and Rolli's versions of Hamlet's famous soliloquy stress different conceptions of 
translation which, in any case, end up acknowledging the sublime genius of Shakespeare's language.

Keywords: Paolo Rolli, Voltaire, Hamlet's Soliloquy, transnational critical Debate, literary Models, Conceptions of Translation. 